\title{
Activadores alternativos para cementos de activación alcalina.
}

\author{
J. Payá ${ }^{1 *}$, J. Monzó ${ }^{1}$, M.V. Borrachero ${ }^{1}$, L. Soriano ${ }^{1}$, M.M. Tashima ${ }^{2}$. \\ *Autor de Contacto: jjpaya@cst.upv.es \\ ${ }^{1}$ Grupo de Investigación en Química de los Materiales (GIQUIMA), Instituto de Ciencia y Tecnología del \\ Hormigón (ICITECH), Universitat Politècnica de València, Valencia, España. \\ ${ }^{2}$ Grupo de Pesquisa MAC- Materiais Alternativos de Construção, Universidade Estadual Paulista (UNESP), \\ Campus de Ilha Solteira, São Paulo, Brasil.
}

\section{RESUMEN}

Los cementos de activación alcalina (CAA) requieren de un componente alcalino para la activación del precursor. La fabricación del activador alcalino (AA) supone un consumo energético y de materias primas muy importante, de modo que la huella de carbono de los CAA está fundamentalmente influenciada por ese factor. Una alternativa es el uso de otras materias para la preparación del AA. En este trabajo se realiza un exhaustivo análisis de las diferentes alternativas: materias de base silícea para la preparación de silicatos alternativos y materias de base alcalina. Se comparan de forma relativa las huellas de carbono con respecto a cementos Portland comerciales, y se analiza el efecto que tiene la sustitución de reactivos comerciales por activadores alternativos.

Palabras clave: Activación alcalina; activadores alternativos; cenizas de biomasa; residuos industriales; huella de carbono 


\begin{abstract}
Alkali activated cements (AAC) require an alkaline component for activating of the precursor. The manufacture of the alkaline activator (AA) involves a very important energy and raw material consumption, so that the carbon footprint $(\mathrm{CF})$ of $\mathrm{AAC}$ is basically influenced by this factor. An alternative is the use of other materials for AA preparation. In this work an exhaustive analysis of the different alternatives is carried out: silica-based materials to prepare of alternative silicates and alkaline-based materials. CF is compared relative to commercial Portland cements, and the effect of replacing commercial reagents with alternative activators is analyzed.
\end{abstract}

Keywords: Alkali activation; alternative activators; biomass ash; industrial waste; carbon footprint

\title{
RESUMO
}

Cimentos ativados alcalinamente (CAA) requerem um componente alcalino para ativação do precursor. A fabricação do ativador alcalino (AA) está associada um consumo de energia e matéria-prima muito importante, de forma que a pegada de carbono do CAA é basicamente influenciada por este fator. Uma alternativa é o uso de outros materiais para a preparação do AA. Neste trabalho é realizada uma análise exaustiva das diferentes alternativas: materiais à base de sílica para a preparação de silicatos alternativos e materiais à base de álcali. As pegadas de carbono são comparadas com os cimentos Portland comerciais e o efeito da substituição dos reagentes comerciais por ativadores alternativos é analisado.

Palavras-chave: Ativação alcalina; ativadores alternativos; cinza de biomassa; resíduo industrial; pegada de carbono

\section{INTRODUCCIÓN}

El término "Economía Circular" se ha instaurado en la mayor parte de las propuestas sobre el desarrollo de la humanidad en los retos globales (cambio climático, biodiversidad, residuos y contaminación). Esta economía circular está enfocada a un modelo productivo+consumo en el que se priorice la reducción, la reutilización, la reparación, el reciclado, la recuperación y la valorización de productos. De este modo, el ciclo de vida de los productos se debe extender en la mayor medida de lo posible, minimizando tanto el consumo de recursos naturales y de energía como la generación de residuos y contaminación del entorno.

La circularidad puede y debe extenderse a cada uno de las áreas de desarrollo, científicas, tecnológicas y humanas. Dado que muchos de los procesos de fabricación están relacionados con procesos y reacciones químicas, del mismo modo que los fenómenos de contaminación y de gestión de residuos, toma especial importancia el término "Química Circular" (Keijer at al., 2019; Mohan, y Katakojwala, 2021). La Química es la ciencia esencial para la Economía Circular, donde la estrategia se enfoca hacia la innovación química a nivel atómico, molecular y estructural.

El área de la construcción y, específicamente, el área del hormigón, el material más utilizado por la humanidad después del agua, deben desarrollarse bajo el paraguas de la Química Circular. El cemento, como material clave conglomerante en la preparación de hormigones, es un material sintético fabricado a partir de diversos componentes y que está constituído por un conjunto de compuestos químicos, que por reacción con el agua desarrollan otros productos químicos que son los responsables de las propiedades tecnológicas de los conglomerantes.

En la antigüedad ya se preparaban los conglomerantes aéreos basados en yeso y cal hidratada a través de la transformación de materias naturales, y dichos conglomerantes reaccionaban con agua 
y/o con dióxido de carbono para producir materiales resistentes y con estabilidad para ciertos entornos. La introducción por los ingenieros romanos de los materiales puzolánicos, fundamentalmente cenizas de origen volcánico, en las mezclas con cal hidratada, dio un giro químico transcendental (Pavía y Caro, 2008), que, muchos siglos más tarde, sería retomado para el desarrollo de nuevos cementos con adiciones puzolánicas.

A finales del siglo XVIII y principios del XIX, se implementaron procesos químicos para la fabricación de conglomerantes hidráulicos, que culminaron con la patente de Joseph Aspdin en 1824: se inició, por tanto, el período del cemento Portland desplazando el uso de materiales como la cal y el yeso. El cemento es el material que conforma desde ese momento hasta nuestros días una gran parte de edificaciones e infraestructuras (Hall, 1976). El desarrollo de procesos químicos a alta temperatura (clinkerización) permitieron la elaboración de cementos de elevadas prestaciones y aceptable durabilidad. El conocimiento de las reacciones químicas de hidratación del cemento Portland y la interacción de éstas con el resto de componentes del hormigón y del entorno (humedad, $\mathrm{CO}_{2}$, cloruros, ...) ha permitido la evolución de las características de los nuevos cementos comerciales basados en el clinker del cemento Portland.

Listado de acrónimos (por orden de aparición en el texto)

\begin{tabular}{|l|l|l|l|}
\hline \multicolumn{2}{|c|}{ En Español } & \multicolumn{2}{c|}{ En inglés } \\
\hline Acrónimo & Significado & Significado & Acrónimo \\
\hline AA & Activador alcalino & Alkali activator & AA \\
\hline CAA & Cemento de activación alcalino & Alkali activated cemnent & AAC \\
\hline MK & Metacaolín & Metakaolin & MK \\
\hline CV & Ceniza volante de carbón & Pulverized fly ash & FA \\
\hline ESC & Escoria de alto horno & Blast furnace slag & BFS \\
\hline CER & Residuo cerámico & Ceramic waste & CW \\
\hline NS & Nanosílice de craqueo & Spent fluid catalytic & FCC \\
\hline TD & Tierra de diatomeas & Nanosílica & DE \\
\hline FCC & Catalizador gastado catyst & WG \\
\hline SS & Satalítico & Sodium silicate & WD \\
\hline TDR & Tierra de diatomeas residual & Wasted diatomite & SF \\
\hline HS & Humo de sílice & Silica fume & GW \\
\hline RV & Residuo de vidrio & Glass waste & RHA \\
\hline CCA & Ceniza de cáscara de arroz & Rice husk ash & SCSA \\
\hline CPCA & Ceniza de paja de caña de azúcar & Sugar cane straw ash & SCBA \\
\hline CBCA & Ceniza de bagazo de caña de azúcar & Sugar cane bagasse ash & BLA \\
\hline CHB & Ceniza de hoja de bamboo & Bamboo leaf ash & BL \\
\hline LB & Licor Bayer & Bayer Liquor & RM \\
\hline LR & Lodo rojo & Red mud & ACS \\
\hline LLA & Líquido limpiador alcalino & Alkaline cleaning solution & OBA \\
\hline CHO & Ceniza de hueso de oliva & Olive-stone biomass ash & ABA \\
\hline CCAL & Ceniza de cáscara de almendra & Almond Shell biomass ash & HCWA \\
\hline CMAC & Ceniza de madera con alto calcio & High calcium wood ash & CCA \\
\hline CMM & Ceniza de mazorca de maíz & Corn cob ash & Foms earth \\
\hline
\end{tabular}

En las últimas décadas, la preocupación por la emisión de los gases de efecto invernadero que supone la fabricación del cemento Portland, el empeño por la reducción en el consumo de materias primas no renovables y los requerimientos en la mejora del desempeño tecnológico de los 
hormigones, ha llevado a la química de los materiales a nuevos caminos más sostenibles, complementarios y más circulares (Phair, 2006). Se ha investigado en profundidad sobre cementos con mayor proporción de adiciones puzolánicas e hidráulicas, cementos belíticos, cementos de sulfoaluminato de calcio, cementos de magnesia y cementos de activación alcalina.

El presente trabajo se centra en los cementos de activación alcalina (CAA), específicamente en los activadores alcalinos (AA) utilizados, su impacto en términos de huella de carbono y en las alternativas que supone una aplicación de la Química Circular. El trabajo está desglosado en una sección inicial sobre conceptos básicos de la química de los cementos de activación alcalina, seguida de una evaluación comparativa de la huella de carbono asociada a los diferentes cementos de activación alcalina convencionales, con respecto a los cementos basados en clinker Portland, para finalizar con una descripción de los materiales alternativos, de origen residual, que pueden ser usados para la elaboración de los activadores alcalinos y la evaluación de la huella de carbono potencial.

\section{NATURALEZA DE LOS CEMENTOS DE ACTIVACIÓN ALCALINA (CAA) Y ORIGEN DE LOS COMPONENTES}

Básicamente, los cementos de activación alcalina (CAA) se forman por la combinación de dos componentes (two part): el mayoritario es el precursor, de base química $\mathrm{SiO}_{2}-\mathrm{Al}_{2} \mathrm{O}_{3}$ (ejemplos: metacaolin $\mathrm{MK}$, ceniza volante de central termoeléctrica de carbón $\mathrm{CV}$ ), aunque en algunos casos también puede contener cantidades significativas de $\mathrm{CaO}$ (escorias de alto horno, $\mathrm{ESC}$ ). El otro componente, minoritario, es una disolución acuosa altamente alcalina (hidróxidos, silicatos, carbonatos de sodio o de potasio). La mezcla de estos dos componentes produce la formación de sustancias de carácter cementante del tipo silicoaluminato alcalino $(\mathrm{Na}, \mathrm{K})$ hidratado $(\mathrm{N}(\mathrm{K})$-A-SH) o, en el caso de presencia de calcio, silicoaluminato cálcico hidratado (C-A-S-H); también se pueden producir geles de naturaleza híbrida entre los dos anteriores, $\mathrm{C}(\mathrm{N}, \mathrm{K})-\mathrm{A}-\mathrm{S}-\mathrm{H}$.

La gran ventaja de estos CAA, desde el punto de vista de la química circular y desde el punto de vista de la sostenibilidad, reside en que, para muchos casos, se pueden usar residuos procedentes de otras actividades antropogénicas de tipo industrial y agrícola. Este es el caso de ESC y de CV, así como residuos de construcción como los de tipo cerámico, CER. En otros casos, la circularidad no es posible puesto que el precursor se fabrica ex profeso, como el MK (calcinación de caolín a $800^{\circ} \mathrm{C}$ ). En los CAA se evita la aplicación de temperaturas muy altas, como ocurre en la clinkerización del cemento Portland $\left(1450^{\circ} \mathrm{C}\right)$.

La gran desventaja de los CAA es que el activador es una sustancia de síntesis química: hidróxidos tales como $\mathrm{NaOH}$ y $\mathrm{KOH}$, carbonatos $\mathrm{Na}_{2} \mathrm{CO}_{3}$ y $\mathrm{K}_{2} \mathrm{CO}_{3}$, silicatos $\mathrm{Na}_{2} \mathrm{SiO}_{3}$ (vidrio soluble, waterglass) y $\mathrm{K}_{2} \mathrm{SiO}_{3}$. La obtención de estas sustancias supone el empleo de recursos naturales, la necesidad de procesos químicos y, sobre todo, un cuantioso consumo de energía, lo que conlleva un impacto significativo y un alejamiento de la circularidad.

En los últimos años ha habido un desarrollo importante de los CAA en los que los dos componentes están combinados en uno solo (one part technology), de modo que su aplicación sigua el mismo modelo que para los cementos tradicionales: adición de agua al cemento "one part".

\section{HUELLA DE CARBONO DE LOS CEMENTOS DE ACTIVACIÓN ALCALINA}

Es bien conocido que la emisión de dióxido de carbono $\left(\mathrm{CO}_{2}\right)$ relacionada con la fabricación del clínker de cemento Portland, es decir, la huella de carbono, es muy elevada: el proceso supone una emisión de 850-1200 $\mathrm{kg} \mathrm{CO} /$ t-clínker, todo ello dependiendo, fundamentalmente, de las tecnologías utilizadas y la efectividad en la recuperación del calor y el aislamiento de los elementos 
industriales. Se considera que la emisión de $\mathrm{CO}_{2}$ tiene dos componentes: por una parte, la componente química, $\mathrm{y}$, por otra, la componente energética.

La componente química es debida a la descarbonatación de la caliza de acuerdo con la siguiente ecuación química (eq 1):

$$
\mathrm{CaCO}_{3} \stackrel{\Delta}{\rightarrow} \mathrm{CaO}+\mathrm{CO}_{2}
$$

Para un clínker que contiene un $64 \%$ de $\mathrm{CaO}$, la cantidad de $\mathrm{CO}_{2}$ de la componente química $\left(\mathrm{CO}_{2} \mathrm{q}\right)$ es de aproximadamente $500 \mathrm{kgCO}_{2} / \mathrm{t}$-clínker. La cantidad de $\mathrm{CO}_{2}$ de la componente de energía $\left(\mathrm{CO}_{2} \mathrm{fd}\right)$, asociada al consumo de combustible y de energía eléctrica, es aproximadamente igual a la de $\mathrm{CO}_{2} \mathrm{q}$ (Luukkonen et al., 2016). A nivel comparativo, podemos establecer un valor de 100 para la emisión de $\mathrm{CO}_{2}$ asociada a un cemento con 95\% de clínker (cemento de tipo CEM I en la nomenclatura europea), con una contribución de 50 para $\mathrm{CO}_{2} \mathrm{q}$ y 50 para $\mathrm{CO}_{2} \mathrm{fd}$ (Ver Figura 1, CEM I). En el caso de cementos con adiciones minerales, se reduce de forma proporcional cada una de las contribuciones: en la Figura 1 se muestran los casos de CEM II/A (con 20\% de adición) y CEM II/B (con 35\% de adición). En la actualidad, a nivel europeo se está trabajando con la normalización del cemento CEM II/C-M (EN 197-5, 2021), en el que existe una mezcla de adiciones (M) hasta un 50\%, ejemplo que también se muestra en la Figura 1. En el caso de los cementos con adiciones, debemos considerar que puede existir un consumo de energía en la preparación de las mismas (tamizado, molienda, secado), bien en procesos independientes o bien en procesos conjuntos de mezcla con el clínker sin moler. Es por ello que, en esos casos, existe una componente adicional que hemos denominado acondicionamiento.

Los activadores alcalinos sintéticos tienen asociados determinados valores de emisión de $\mathrm{CO}_{2}$ equivalente $\left(\mathrm{CO}_{2}\right.$-e, $\mathrm{kgCO}_{2} / \mathrm{kg}$-reactivo); dichos valores dependen mucho del proceso industrial y de la tecnología usada en la síntesis (ver Tabla 1). Así, por ejemplo, el $\mathrm{NaOH}$ se puede obtener por medio de la caustificación (eq 2) o por electrolisis del cloruro sódico (eq 3):

$$
\begin{gathered}
\mathrm{Ca}(\mathrm{OH})_{2}+\mathrm{Na}_{2} \mathrm{CO}_{3} \rightarrow \mathrm{CaCO}_{3}+2 \mathrm{NaOH} \\
\mathrm{NaCl}+2 \mathrm{H}_{2} \mathrm{O} \rightarrow \mathrm{NaOH}+\mathrm{Cl}_{2}+\mathrm{H}_{2}
\end{gathered}
$$

Los CAA basados en metakaolín (MK) requieren de cantidades significativas de activador, sobre todo de silicato sódico SS (Weil et al., 2009). Asimismo, la fabricación de MK requiere de la calcinación a $800^{\circ} \mathrm{C}$ del caolín, por lo que la síntesis del precursor conlleva un importante consumo de energía. Un cálculo aproximado de las emisiones asociadas para un CAA basado en MK se muestra en la Figura 2: puede observarse que, comparando con CEM I, no existe una diferencia significativamente ventajosa. Lógicamente, será tanto más ventajosa cuanto menor sea el consumo de silicato sódico en la dosificación. Los CAA basados en ceniza volante $(\mathrm{CV})$ también requieren de cantidades muy importantes de activador, y para conseguir un buen desarrollo de la geopolimerización se necesita un curado a elevada temperatura, por lo que existe un consumo energético adicional a tener en cuenta en su aplicación (curado). En la Figura 2 se muestra que la emisión de $\mathrm{CO}_{2}$ asociada es también muy elevada.

En los dos casos anteriores, basados en $\mathrm{MK}$ y $\mathrm{CV}$, el contenido en calcio de dichos precursores es bajo, lo que requiere de cantidades elevadas de activador. En el caso de precursores ricos en calcio, como en la escoria de alto horno ESC, se requiere de menores cantidades de activador. Además, se puede activar el precursor solamente con $\mathrm{NaOH}$, sin la necesidad de usar silicato alcalino (SS o silicato de potasio). En la Figura 2 se muestra la gran ventaja existente para CAA-ESC con respecto a la huella de carbono relativa al compararla con los anteriores CAA comentados. La escoria requiere de la molienda previa (acondicionamiento precursor) para poder facilitar la reactividad en el medio alcalino. 


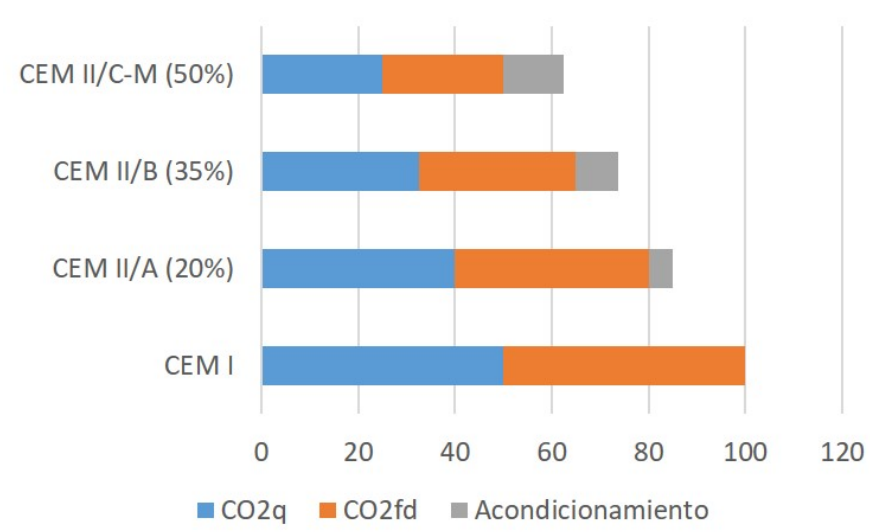

Clave:

$\mathrm{CO}_{2} \mathrm{q}$ : componente química

$\mathrm{CO}_{2} \mathrm{fd}$ : componente energética; Acondicionamiento: tratamiento de las adiciones, previo o simultáneo con el clinker).

Figura 1: Comparativa de emisiones relativas de cementos basados en clínker de cemento Portland: CEM I, CEM II/A (con 20\% de adición), CEM II/B (con 35\% de adición) y CEM II/CM (con 50\% de adición). Escala relativa tomando 100 para el CEM I.

Tabla 1. Emisiones de $\mathrm{CO}_{2}$ asociadas $\left(\mathrm{CO}_{2}\right.$-e, $\mathrm{kgCO}_{2} / \mathrm{kg}$-reactivo $)$ de algunos reactivos alcalinos

\begin{tabular}{|c|c|}
\hline Reactivo & $\mathrm{CO}_{2}$-e \\
\hline $\mathrm{NaOH}$ & 1.12 \\
\hline $\mathrm{KOH}$ & 1.94 \\
\hline $\mathrm{Na}_{2} \mathrm{CO}_{3}$ & 0.59 \\
\hline $\mathrm{K}_{2} \mathrm{CO}_{3}$ & 2.38 \\
\hline $\mathrm{Na}_{2} \mathrm{SiO}_{3}$ & 1.64 \\
\hline
\end{tabular}

Tomado de: https://www.winnipeg.ca/finance/findata/matmgt/documents/2012/ 682-2012/6822012_Appendix_H-

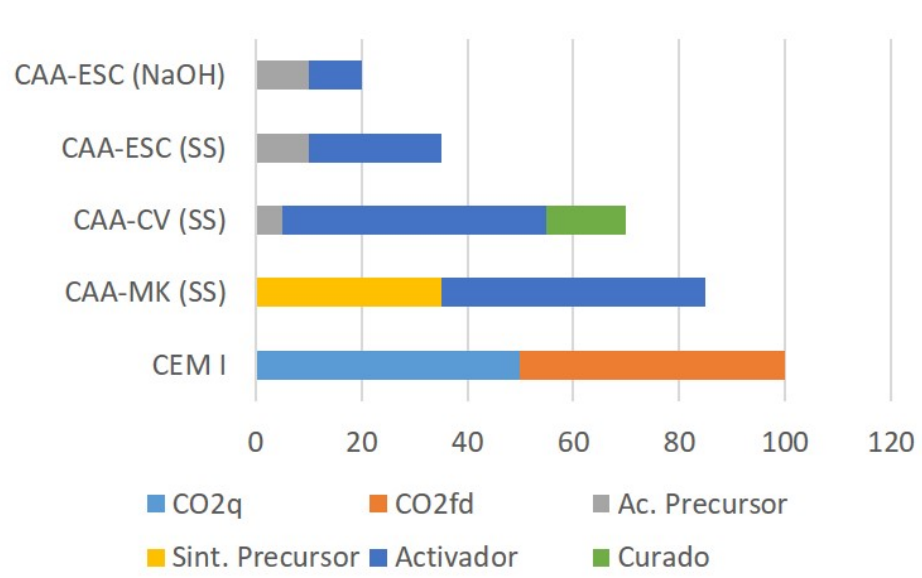

Clave:

$\mathrm{CO}_{2} \mathrm{q}$ : componente química $\mathrm{CO}_{2} \mathrm{fd}$ : componente energética

Ac. precursor: Acondicionamiento (molienda)

Sint. Precursor: proceso de síntesis del precursor (calcinación)

Activador: asociado a la fabricación del activador

Curado: asociado al consumo de energía para el curado).

Figura 2: Comparativa de emisiones relativas de cementos CEM I con los CAA basados en metacaolín (MK), ceniza volante (CV) y escoria de alto horno (ESC). Activadores: hidróxido sódico $(\mathrm{NaOH})$ y silicato sódico $(\mathrm{SS})$. Escala relativa tomando 100 para el CEM I.

\section{ACTIVADORES ALCALINOS ALTERNATIVOS}

Como se ha podido comprobar en la sección anterior, la contribución del activador a la huella de carbono del CAA es muy relevante, por lo que las mejoras a este respecto deben centrarse en minimizar este componente. Una opción se focalizaría en la mejora de los procesos químicos de síntesis de los reactivos químicos, de modo que se reduzca el $\mathrm{CO}_{2}$-e asociado. La otra opción, más 
adecuada para la economía y química circulares, es la valorización de residuos de carácter agrícola e industrial.

En los últimos años, se ha venido produciendo una escalada en la publicación de artículos científicos en los que se valorizan residuos, con el objetivo de conseguir activadores eficientes que tengan una menor huella de carbono. En estas publicaciones podemos encontrar propuestas variadas, y que podríamos clasificar en los siguientes conjuntos:

a) Activadores alternativos basados en sílice: son activadores relacionados con los silicatos alcalinos, donde se hace reaccionar un hidróxido alcalino con una materia prima rica en sílice, la cual se disuelve total o parcialmente.

b) Activadores alternativos basados en álcalis: en este caso, el propio material usado es ya propiamente una sustancia alcalina, de base sódica o potásica.

c) Sistemas de activadores combinados sílice-álcali.

\subsection{Activadores alternativos basados en sílice.}

En este conjunto podemos encontrar distintas estrategias; por una parte, el uso de recursos naturales de base silícea que puedan ser disueltos en diferentes condiciones; por otra parte, disponemos de cenizas procedentes de la combustión de biomasa, cenizas con elevados porcentajes de $\mathrm{SiO}_{2}$; $\mathrm{y}$, finalmente, podemos usar algunos residuos industriales ricos en sílice potencialmente reactiva.

\subsubsection{Activadores de base silícea procedentes de recursos naturales.}

En este apartado, encontramos referencias de dos recursos: la tierra de diatomeas y el olivino.

La tierra de diatomeas (TD) es un material rico en sílice (generalmente $\mathrm{SiO}_{2}>80 \%$ ) formado por los esqueletos de diatomeas, algas unicelulares que se han depositado a lo largo de millones de años. Esta roca de origen sedimentario presenta sílice en estado amorfo, la cual puede ser disuelta en condiciones alcalinas. Font et al. (2018) presentaron un estudio en el que se hacía reaccionar TD con $\mathrm{NaOH}$ en un recipiente aislado térmicamente: la disolución de los pellets de $\mathrm{NaOH}$ en agua aumenta la temperatura del medio acuoso y eso favorece la disolución de la sílice amorfa. Los resultados obtenidos muestran un buen comportamiento del activador preparado. Así, estos autores usaron este activador para el precursor FCC (catalizador usado de craqueo catalítico), obteniendo, en morteros, resistencias mecánicas de $30 \mathrm{MPa}$ a los 7 días de curado a temperatura ambiente, sin necesidad de un curado a alta temperatura.

El olivino, $(\mathrm{Fe}, \mathrm{Mg})_{2} \mathrm{SiO}_{4}$, es una materia natural que puede ser usada para la obtención de nanosílice, a partir de un proceso de disolución con ácido sulfúrico concentrado y posterior filtración (Gao et al., 2017). Estos autores usaron una disolución preparada con nanosílice (NS) y $\mathrm{NaOH}$, y demostraron que la reactividad fue similar a la del silicato sódico comercial (waterglass, SS). Aplicaron este activador a una mezcla de escoria de alto horno (ESC) y de ceniza volante (CV), en una proporción 70/30, y la resistencia mecánica de los hormigones obtenidos se encontró en el intervalo 57-68 MPa a 7 días y 72-82 MPa a los 28 días, dependiendo del módulo de sílice (relación molar $\mathrm{SiO}_{2} / \mathrm{Na}_{2} \mathrm{O}$ ) utilizado en la activación.

\subsubsection{Activadores de base silícea procedentes de residuos industriales.}

En este apartado describiremos ejemplos basados en tierra de diatomeas residuales, humo de sílice y residuos de vidrio.

La tierra de diatomeas se usa frecuentemente como componente de materiales filtrantes de líquidos, especialmente cerveza y vino. Al final del proceso de filtración se genera una tierra de diatomeas residual (TDR) que sigue presentando cantidades importantes de sílice amorfa potencialmente soluble. La TDR procedente del filtrado de cerveza (TDR-C) se usó para preparar activadores con $\mathrm{NaOH}$ (Mejía et al., 2016); este activador se usó con un precursor formado por una mezcla de $\mathrm{CV}$ y MK (70/30) y la pasta se curó a $60^{\circ} \mathrm{C}$ durante 24 horas y posteriormente, se curó a temperatura 
ambiente hasta los 360 días. Los valores de resistencia a compresión en el intervalo 7-360 días estuvieron cercanos a los $35 \mathrm{MPa}$. La TDR procedente del filtrado del vino (TDR-V) fue estudiada por Font et al. (2018); estos autores evaluaron el comportamiento del activador basado en este residuo y observaron que se producía una mejora sustancial si la TDR-V se calcinaba a $650^{\circ} \mathrm{C}$ para eliminar la materia orgánica procedente de la filtración: la resistencia mecánica a compresión, a los 28 días, de morteros de FCC activado era de unos $20 \mathrm{MPa}$, para el sistema sin la calcinación previa de la TDR-V, mientras que alcanzaba los $40 \mathrm{MPa}$ para el sistema con la calcinación.

El humo de sílice (HS) es bien conocido por ser una puzolana excelente en los sistemas con cemento Portland, dada la elevada finura y el carácter amorfo de esa adición mineral de base silícea. Este residuo, procedente de la industria del silicio y las aleaciones de ferrosilicio, puede ser usado como materia prima para la elaboración de activadores. Así, Rouseková et al. (1997) estudiaron la activación de ESC con una mezcla de $\mathrm{HS}$ y $\mathrm{NaOH}$, obteniendo buenos resultados. Posteriormente, Bernal et al. (2012) estudiaron el activador $\mathrm{HS} / \mathrm{NaOH}$ en sistemas $\mathrm{MK}+\mathrm{ESC}$; demostraron que la sílice proveniente del HS era incorporada a tiempos muy cortos en la reacción de geopolimerización, y se formaban mezclas de geles cementantes C-A-S-H, N-A-S-H, o incluso gel $\mathrm{N}-(\mathrm{C})-\mathrm{A}-\mathrm{S}-\mathrm{H}$.

En cuanto a los residuos de vidrio (RV), podemos encontrar diversas composiciones químicas de los mismos, aunque lo más destacable es el estado amorfo y un elevado porcentaje de sílice. Desde 2014 se ha venido estudiando el potencial de RV para fabricar activadores. Los porcentajes de $\mathrm{SiO}_{2}$ en RV están mayoritariamente en el intervalo 65-80\%, y contenidos significativos de $\mathrm{CaO}_{\text {y }} \mathrm{Na}_{2} \mathrm{O}$ (Liu et al., 2019). Se han realizado diversas aproximaciones para la preparación del activador alcalino, como se muestra en la Figura 3: método de fusión, métodos hidrotermales y método termoquímico. Se ha demostrado que la naturaleza y velocidad de formación de los hidratos cementantes depende, fundamentalmente, del tipo de precursor.

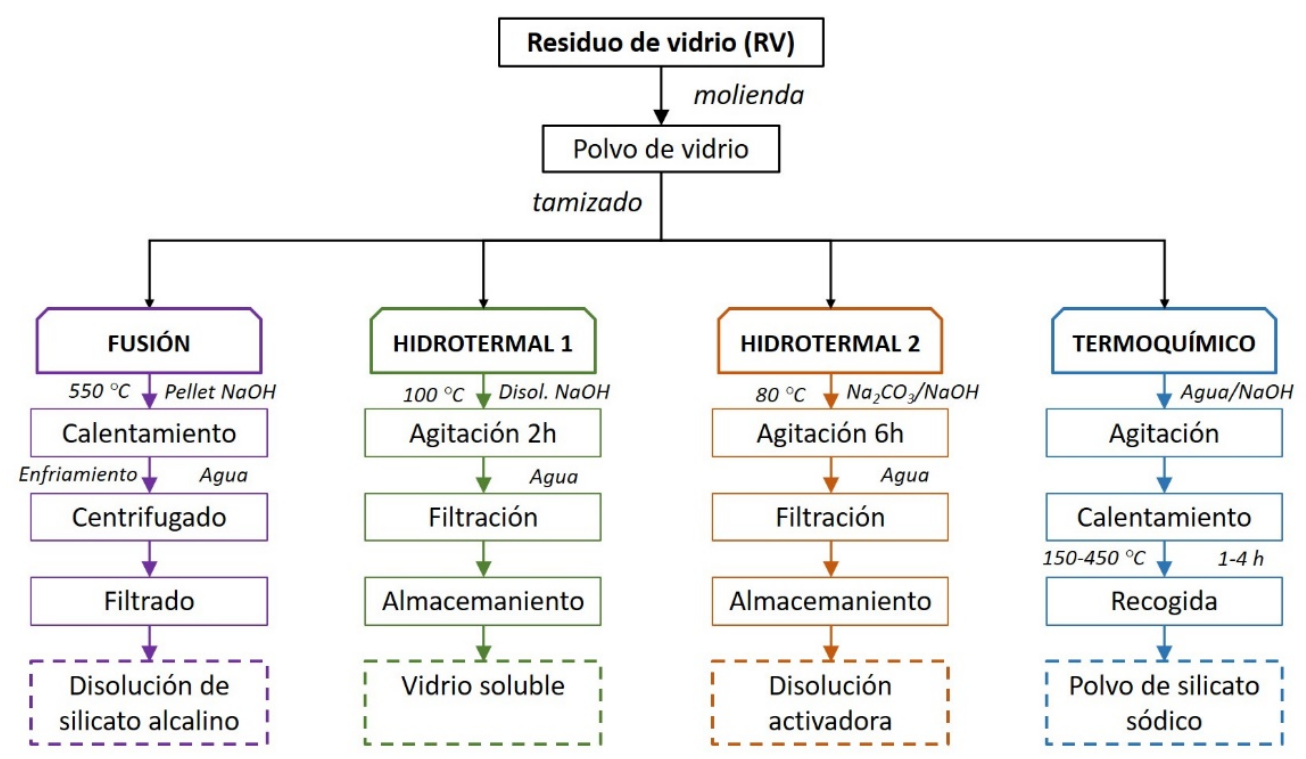

Figura 3: Métodos para la obtención de activadores alternativos a partir de residuo de vidrio (RV). (Adaptado de Lie et al, 2019).

\subsubsection{Activadores de base silícea procedentes de cenizas de biomasa.}

En este apartado se encuentra el mayor número de ejemplos de activadores alternativos. La combustión de biomasa, en algunos casos, genera la formación de una cantidad relativamente importante (1-4\% en masa) de cenizas. Los ejemplos más significativos son los de la cáscara de arroz, los residuos procedentes del cultivo y procesado de la caña de azúcar, y la hoja de bambú (Roselló et al., 2015). 
El caso de la ceniza obtenida de la cáscara de arroz (CCA) es el más estudiado. Se ha publicado un número importante de ejemplos con el uso de CCA para la fabricación de silicato sódico. La primera referencia bibliográfica se remonta a 2010 (Bejarano et al., 2010); los autores proponen un proceso hidrotermal en el que analizan diferentes variables: la relación $\mathrm{NaOH} / \mathrm{CCA}$, el tiempo de tratamiento, la temperatura de reacción y la proporción agua/CCA. La conclusión sobre los parámetros óptimos eran $\mathrm{NaOH} / \mathrm{CCA}=2$, agua $/ \mathrm{CCA}=10,100^{\circ} \mathrm{C}$ de tratamiento por 120 minutos. Con estas proporciones, este grupo de investigación usó este tipo de activador en la activación alcalina de diversos precursores habituales (Mejía et al., 2016). Bouzón et al (2014) aplicaron un activador obtenido por reflujo de una mezcla de disolución de $\mathrm{NaOH}$ con CCA parcialmente amorfa, usando FCC como precursor en la preparación del mortero. Se obtuvieron sistemas con excelentes prestaciones mecánicas, si bien se observó que el activador era más reactivo si se usaba CCA molida en el proceso hidrotermal (alrededor de 60 minutos, frente a los 120 minutos para la muestra no molida). Otros autores han profundizado en métodos y aplicaciones (entre otros: Tong et al., 2018; Rajan and Kathirvel, 2021). Villca et al. (2021) han usado un activador alternativo basado en CCA, fabricado sin consumo de energía; simplemente se ha usado el calor de disolución de los pellets de $\mathrm{NaOH}$ para disolver la sílice amorfa de CCA. Este activador fue aplicado, por primera vez, en sistemas binarios de cal/puzolana y geopolímero.

El cultivo de la caña de azúcar produce un residuo, la paja de la caña, que habitualmente es abandonada en el campo. El uso de esta biomasa podría repercutir en un beneficio, en cuanto a la valorización energética. El residuo generado, la ceniza de paja de caña de azúcar (CPCA) contiene cantidades elevadas de sílice (Moraes et al., 2018). En esta investigación, se demostró que el procedimiento que aprovecha el calor de disolución del $\mathrm{NaOH}$ para disolver la sílice es perfectamente viable para CPCA, y que el tratamiento no debe superar las 24 horas, porque se produce una gelificación del activador. La ceniza de bagazo de caña de azúcar (CBCA) también se usó (Tchakouté et al., 2017) en la geopolimerización de MK; el conglomerante preparado alcanzó los $33 \mathrm{MPa}$ a los 28 días de curado.

Finalmente, la ceniza de hoja de bambú (CHB), que posee un porcentaje de $\mathrm{SiO}_{2}$ cercano al $80 \%$ (Roselló et al., 2015), se constituye como otra opción interesante. La CHB se usó a través de un proceso termoquímico (Figura 3) con una temperatura de $300-330^{\circ} \mathrm{C}$ (Vinai et al., 2021). El activador se usó para el sistema CV/ESC (60/40) y se ha comprobado que, si bien, comparado con el activador $\mathrm{SS}+\mathrm{NaOH}$, la reactividad es menor en los primeros 7 días de curado, a los 28 días se alcanzan las mismas resistencias (alrededor de $43 \mathrm{MPa}$ ).

\subsection{Activadores alternativos basados en álcalis.}

La actividad humana también produce residuos de carácter alcalino, tanto de origen industrial como de origen agrícola.

\subsubsection{Activadores de base alcalina procedentes de actividad industrial.}

Entre ellos podemos encontrar algunos residuos relacionados con la actividad metalúrgica, tales como el licor Bayer, los líquidos limpiadores de metales y los lodos rojos.

El licor Bayer (LB) es una disolución cáustica preparada con $\mathrm{NaOH}$ y que se usa para la extracción del aluminio de la bauxita. La solución resultante, después del filtrado, es reutilizable, pero finalmente hay que descartarla cuando disminuye la eficiencia de la misma. Ese residuo, rico en aluminato sódico, se ha usado para la activación de la CV en diferentes condiciones (Jamieson et al. 2016).

El lodo rojo (LR) es el residuo sólido que se genera en la extracción del aluminio de la bauxita, y es muy rico en óxidos de hierro y sílice. Este lodo tiene un $\mathrm{pH}$ entre 9.7 y 12.8 , y se ha usado para activar CV (Choo et al., 2016), aunque la resistencia alcanzada no superaba los $2 \mathrm{MPa}$. 
El último ejemplo de esta categoría lo constituyen los líquidos limpiadores alcalinos (LLA), que se suelen producir como residuos en la limpieza de superficies de aluminio, de modo que son ricos en hidróxido y aluminato sódicos. Fernández-Jiménez et al. (2017) usaron LLA, con una concentración en $\mathrm{NaOH}$ aproximadamente de $5 \mathrm{M}$, para activar $\mathrm{CV}$ molida y polvo de $\mathrm{RV}$. En ambos casos se pudo obtener resistencias mecánicas significativas, que rondaban los 10-16 $\mathrm{MPa}$ para CV y 2-10 MPa para RV (curados durante 20 horas y $85^{\circ} \mathrm{C}$ ).

\subsubsection{Activadores de base alcalina procedentes de residuos agrícolas.}

Algunos residuos agrícolas son biomasas valorizables para la obtención de energía y sus cenizas resultantes tienen carácter alcalino. Ese carácter alcalino viene determinado por la presencia de calcio y, especialmente, de potasio. Este es el caso de la ceniza de hueso de oliva (CHO), de la ceniza de cáscara de almendra (CCAL) y de la ceniza de mazorca de maíz (CMM), así como de cenizas procedentes de madera con alto contenido en calcio (CMAC). La gran ventaja del uso de estas cenizas es que permiten la preparación de cementos "one part", ya que son materiales sólidos. El primer estudio sobre el uso de CHO en CAA fue presentado por Font et al. (2017); se activó ESC con $\mathrm{CHO}$ y se comparó con la activación por medio del reactivo químico $\mathrm{KOH}$. El resultado mostró que la resistencia mecánica a compresión para el sistema ESC/CHO fue superior (29.9 MPa frente a 16.9 MPa). Estudios posteriores (De Moraes Pinheiro et al., 2018) mostraron la formación de geles cementantes del tipo $\mathrm{C}(\mathrm{K})-\mathrm{S}-\mathrm{H}, \mathrm{C}(\mathrm{K})-\mathrm{S}-\mathrm{A}-\mathrm{H}$, además de pequeñas cantidades de hidrotalcita. Posteriormente, Alonso et al. (2019) estudiaron cenizas procedentes de biomasa de olivo, tanto cenizas volantes como cenizas de parrilla: observaron que la alcalinidad de estas cenizas no era suficiente para activar CV pero sí para activar ESC. Payá et al. (2019) emplearon el sistema ESC/CHO para la fabricación de bloques de tierra compactada de base dolomítica. Se obtuvo una resistencia a compresión de bloques compactados de unos $12 \mathrm{MPa}$ a los 9 días con un curado bajo film de plástico (para evitar la evaporación de agua y mantener las condiciones óptimas de hidratación). Los bloques resultantes mostraron excelente resistencia bajo el agua.

Soriano et al. (2020) fueron los primeros en estudiar la potencialidad de ceniza de cáscara de almendra (CCAL). Encontraron que el porcentaje en $\mathrm{K}_{2} \mathrm{O}$ para CCAL era mayor que para $\mathrm{CHO}$ (46.98\% vs. $32.16 \%$ ), y detectaron la presencia de varios minerales de carbonato de calcio y potasio $\left(\mathrm{K}_{2} \mathrm{Ca}\left(\mathrm{CO}_{3}\right)_{2}\right)$, tales como fairchildita y bütschilita, además de calcita $\left(\mathrm{CaCO}_{3}\right)$, portlandita $\left(\mathrm{Ca}(\mathrm{OH})_{2}\right)$ y arcanita $\left(\mathrm{K}_{2} \mathrm{SO}_{4}\right)$. Esta ceniza ofreció un buen rendimiento cuando se combinaba con ESC.

Varios autores han investigado el uso de cenizas de madera con elevado contenido en calcio (CMAC); entre ellos destaca el realizado por Ban et al. (2014), en el que caracteriza la ceniza y la usa como activador sólido para la $\mathrm{CV}$. La ceniza contenía una elevada proporción de $\mathrm{CaO}(61 \%)$ frente a una menor cantidad de $\mathrm{K}_{2} \mathrm{O}(12 \%)$; las sales cálcicas encontradas fueron calcita, portlandita e hidroxiapatito.

También se ha usado la ceniza de mazorca (raquis o coronta) de maíz (CMM) para la activación de MK (Peys et al., 2016): se alcanzó $30 \mathrm{MPa}$ de resistencia a compresión para muestras con una relación $\mathrm{CMM} / \mathrm{MK}$ de 1.2 .

\subsection{Sistemas combinados sílice-álcali.}

Los CAA basados en el uso de cenizas alcalinas permite reducir el impacto, debido a que en algunos casos tanto el precursor como el activador proceden de residuos. Sin embargo, se observa que, frecuentemente, las prestaciones mecánicas no son muy elevadas por el déficit de sílice del activador. En este sentido, el desarrollo de activadores que contengan sílice y álcali permitiría minimizar este efecto. Font et al. (2020) diseñaron CAA con el sistema ternario ESC-CHO-CCA, en el que el activador se preparaba por reacción hidrotermal entre $\mathrm{CCA}$ y $\mathrm{CHO}$, a $65^{\circ} \mathrm{C}$ durante 24 
horas. Para una dosificación ESC-CHO-CCA de 9/4.5/1, se obtuvieron 35.0, 46.2 y 67.4 MPa, para morteros curados durante 7,28 y 90 días, respectivamente, a $20^{\circ} \mathrm{C}$.

\section{HUELLA DE CARBONO DE CEMENTOS DE ACTIVACIÓN ALCALINA CON ACTIVADORES ALTERNATIVOS}

La activación de la escoria con activadores sintéticos supone unas emisiones de $\mathrm{CO}_{2}$ relativamente bajas (Figura 2). Sin embargo, se puede mejorar este aspecto cuando se trabaja con activadores alternativos. Como ejemplo, tomamos el sistema con ceniza de hueso de oliva (CHO). Además de la valorización de residuos (en el caso de la biomasa, también valorización energética) se obtiene una ligera disminución del $\mathrm{CO}_{2}$ asociado. En el caso del sistema ESC-CHO (Figura 4), lógicamente el acondicionamiento del precursor es el mismo, y solamente hay que tener en cuenta el acondicionamiento del activador (en este caso la molienda). Las emisiones de $\mathrm{CO}_{2}$ asociadas para el sistema ESC-CHO-RHA supone valores mayores, puesto que se debe acondicionar el activador por medio del tratamiento hidrotermal de la mezcla CHO-RHA. Pero este nuevo activador permite obtener mayores resistencias mecánicas, por lo que la emisión extra de $\mathrm{CO}_{2}$ puede verse compensada por las prestaciones del material obtenido.

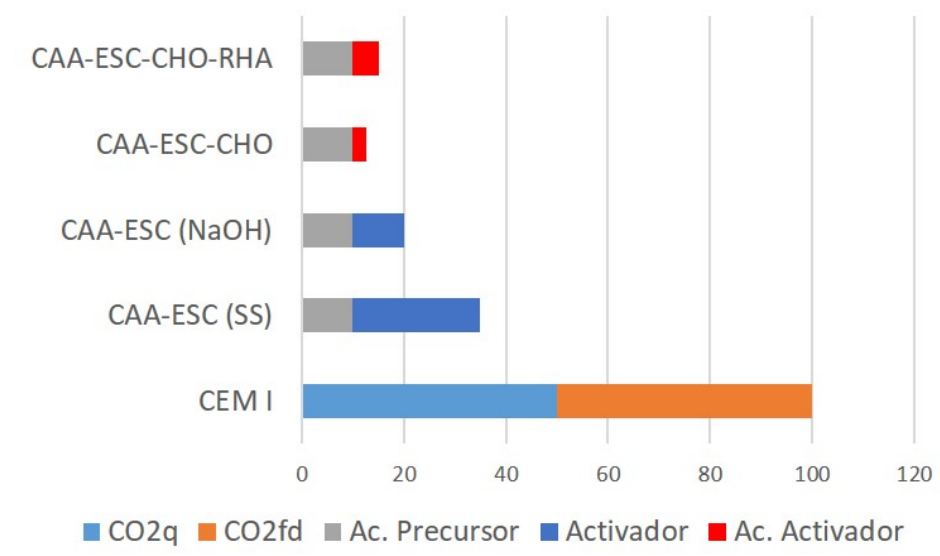

Clave:

$\mathrm{CO}_{2} \mathrm{q}$ : componente química

$\mathrm{CO}_{2} \mathrm{fd}$ : componente energética

Ac. precursor: Acondicionamiento (molienda)

Activador: asociado a la fabricación del activador

Ac. Activador: acondicionamiento de activador (hidrotermal).

Figura 4: Comparativa de emisiones relativas de cementos CEM I con los CAA producidos con activares alternativos sobre precursor de escoria de alto horno (ESC). Activadores comerciales: hidróxido sódico $(\mathrm{NaOH})$ y silicato sódico $(\mathrm{SS})$; Activadores alternativos, ceniza de hueso de oliva $(\mathrm{CHO})$ y ceniza de cáscara de arroz (CCA). Escala relativa tomando 100 para el CEM I.

\section{COMENTARIOS FINALES Y CONCLUSIONES}

Es evidente que el desarrollo de los CAA supone disponer tanto de precursores adecuados como de activadores. Probablemente, no existe a día de hoy suficiente capacidad para disponer de precursores en cantidad y localización suficientes para la sustitución del cemento Portland de forma completa. Por ello, es difícil plantear que los CAA sean una solución excluyente, sino que su producción hay que enfocarla desde el punto de vista de la complementariedad con los cementos Portland y otros tipos de cementos (belíticos, de sulfoaluminato de calcio, de magnesio). El hecho importante es que hay algunos entornos en donde se generan precursores potencialmente comercializables (cerámicos, residuos de construcción, diferentes tipos de escorias metalúrgicas), y deben aprovecharse todas las posibilidades. Lo mismo ocurre con los activadores alternativos: el uso de reactivos químicos de síntesis se puede sustituir en entornos en donde existan actividades agrícolas o industriales que puedan generar componentes adecuados para la preparación del activador. Esta circunstancia puede ser de especial interés en sociedades en vías de desarrollo en 
las que existe dificultad (básicamente económica) para adquirir cemento Portland, pero que disponen de recursos propios para la fabricación de CAA (residuos de construcción, biomasas, ...). Como conclusiones a esta presentación del estado del arte sobre los activadores alternativos, destacan las siguientes:

a) Los CAA no siempre suponen una reducción drástica de las emisiones de $\mathrm{CO}_{2}$ asociadas: hay que considerar que en algunos casos las cantidades necesarias de reactivos químicos suponen una huella de carbono comparable con los cementos Portland.

b) Los precursores como la ESC requieren de cantidades menores de activador, lo que tiene como consecuencia que las emisiones de $\mathrm{CO}_{2}$ sean menores que para el caso de otros precursores como pueden ser la $\mathrm{CV}$ o el MK.

c) Los activadores alternativos pueden ser de origen agrícola o industrial, pero en cualquier caso requieren de una caracterización completa, que integre tanto los estudios de acondicionamiento previo necesario como los potenciales problemas de su uso (presencia de componentes químicos dañinos para algunas aplicaciones, comopor ejemplo, la presencia de cloruros).

d) Los residuos de base silícea no pueden ser usados de forma independiente, debido a su elevada acidez (sílice, fundamentalmente). Los residuos de base alcalina sí pueden ser usados de forma independiente. La combinación de ambos tipos de residuos puede mejorar el comportamiento mecánico de los CAA y puede potenciar sus usos.

e) El uso de cenizas procedentes de biomasa es una alternativa interesante para la síntesis de activadores sostenibles. En el diagrama ternario de la Figura 5 se muestra un esquema modificado del diagrama de Vassilev (Vassilev et al., 2010) sobre la clasificación de las cenizas de biomasa, en la que se han modificado las zonas de reactividad y se han representado diferentes cenizas comentadas en este artículo.

f) El uso de residuos sólidos como activadores alternativos permite la elaboración de cementos "one part". Adicionalmente, se puede realizar una molienda conjunta de precursor y activador lo que mejora la mezcla íntima de ambos componentes.

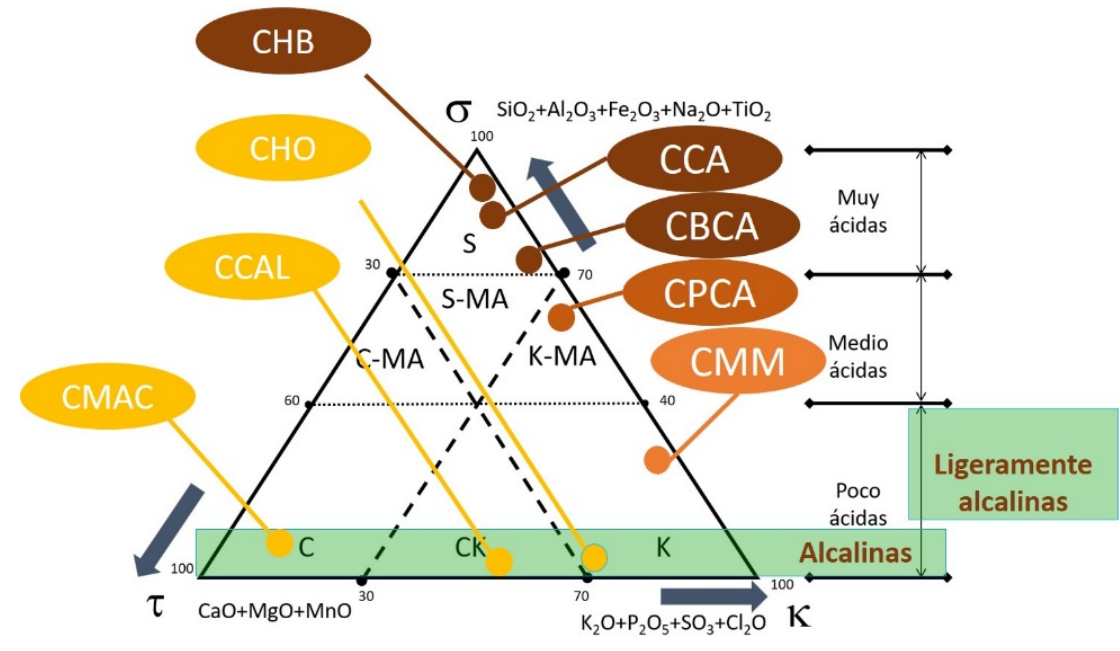

$$
\begin{gathered}
\text { Clave: } \\
\text { ceniza de hueso de oliva (CHO); } \\
\text { ceniza de cáscara del almendra } \\
\text { (CCAL); } \\
\text { ceniza de madera de alto } \\
\text { contenido en calcio (CMAC); } \\
\text { ceniza de mazorca de maíz } \\
\text { (CMM); } \\
\text { ceniza de paja de caña de azúcar } \\
\text { (CPCA); } \\
\text { ceniza de bagazo de caña de } \\
\text { azúcar (CBCA); } \\
\text { ceniza de cáscara de arroz } \\
\text { (CCA); } \\
\text { ceniza de hoja de bambú (CHB) }
\end{gathered}
$$

Figura 5. Diagrama de Vassilev modificado (adaptado de Vassilev et al., 2010). (

\section{AGRADECIMIENTOS}

Por la financiación, al Gobierno de España, Ministerio de Economía y Competitividad (Proyecto BIA2017-87573-C2-1-P) y a los fondos FEDER. 


\section{REFERENCIAS}

Alonso, M.M., Gascó, C., Morales, M.M., Suárez-Navarro, J.A., Zamorano, M., Puertas, F., 2019. Olive biomass ash as an alternative activator in geopolymer formation: A study of strength, durability, radiology and leaching behaviour. Cem. Concr. Compos. 104, 103384. https://doi.org/10.1016/j.cemconcomp.2019.103384

Ban, C.C., Nordin, N.S.A., Ken, P.W., Ramli, M., Hoe, K.W., 2014. The high volume reuse of hybrid biomass ash as a primary binder in cementless mortar block. Am. J. Appl. Sci. 11, 13691378. https://doi.org/10.3844/ajassp.2014.1369.1378

Bejarano, J., Garazón, C., Mejía de Gutiérrez, R., Delvasto, S., Gordillo, M., 2010. In: II Simposio Aprovechamiento de residuos agro-industriales como fuente sostenible de materiales de construcción, Valencia, Spain, November 8-9, 2010

Bernal, S.A., Rodríguez, E.D., Mejia De Gutiérrez, R., Provis, J.L., Delvasto, S., 2012. Activation of metakaolin/slag blends using alkaline solutions based on chemically modified silica fume and rice husk ash. Waste Biomass Valor. 3, 99-108. https://doi.org/10.1007/s12649-011-9093-3

Bouzón, N., Payá, J., Borrachero, M.V., Soriano, L., Tashima, M.M., Monzó, J., 2014. Refluxed rice husk ash/ $\mathrm{NaOH}$ suspension for preparing alkali activated binders. Mater. Lett. 115, 72-74. https://doi.org/10.1016/j.matlet.2013.10.001

Choo, H., Lim, S., Lee, W., Lee, C., 2016. Compressive strength of one-part alkali activated fly ash using red mud as alkali supplier. Constr. Build. Mater. 125, 21-28. https://doi.org/10.1016/j.conbuildmat.2016.08.015

De Moraes Pinheiro, S.M., Font, A., Soriano, L., Tashima, M.M., Monzó, J., Borrachero, M.V., Payá, J., 2018. Olive-stone biomass ash (OBA): An alternative alkaline source for the blast furnace slag activation. Constr. Build. Mater. 178, 327-338. https://doi.org/10.1016/j.conbuildmat.2018.05.157

Fernández-Jiménez, A., Cristelo, N., Miranda, T., Palomo, A., 2017. Sustainable alkali activated materials: Precursor and activator derived from industrial wastes. J. Clean. Prod. 162, 1200-1209. https://doi.org/10.1016/j.jclepro.2017.06.151

Font, A., Soriano, L., Moraes, J.C.B., Tashima, M.M., Monzó, J., Borrachero, M.V., Payá, J., 2017. A $100 \%$ waste-based alkali-activated material by using olive-stone biomass ash (OBA) and blast furnace slag (BFS). Mater. Lett. 203, 46-49. https://doi.org/10.1016/j.matlet.2017.05.129

Font, A., Soriano, L., Reig, L., Tashima, M.M., Borrachero, M.V., Monzó, J., Payá, J., 2018. Use of residual diatomaceous earth as a silica source in geopolymer production. Mater. Lett. 223, 1013. https://doi.org/10.1016/j.matlet.2018.04.010

Font, A., Soriano, L., de Moraes Pinheiro, S.M., Tashima, M.M., Monzó, J., Borrachero, M.V., Payá, J., 2020. Design and properties of 100\% waste-based ternary alkali-activated mortars: Blast furnace slag, olive-stone biomass ash and rice husk ash. J. Clean. Prod. 243, 118568. https://doi.org/10.1016/j.jclepro.2019.118568 
EN 197-5, 2021. Cement - Part 5: Portland-composite cement CEM II/C-M and Composite cement CEM VI.

Gao, X., Yu, Q.L., Lazaro, A., Brouwers, H.J.H., 2017. Investigation on a green olivine nano-silica source based activator in alkali activated slag-fly ash blends: Reaction kinetics, gel structure and $\begin{array}{llllll}\text { carbon footprint. } & \text { Cem. } & \text { Concr. } & \text { Res. } & 100, & 139\end{array}$ https://doi.org/10.1016/j.cemconres.2017.06.007

Hall, C., 1976. On the history of portland cement after 150 years. Journal of Chemical Education, 53(4), 222.

Jamieson, E., van Riessen, A., McLellan, B., Penna, B., Kealley, C., Nikraz, H., 2017. Introducing Bayer liquor-derived geopolymers. In: Handbook of low carbon concrete. Nazari, A. and Sanjayan, J.G. (eds), Kidlington, Oxford, United States: Elsevier.159-193. https://doi.org/10.1016/B978-012-804524-4.00008-7

Keijer, T., Bakker, V. \& Slootweg, J.C., 2019. Circular chemistry to enable a circular economy. Nature Chem 11, 190-195. https://doi.org/10.1038/s41557-019-0226-9

Liu, Y., Shi, C., Zhang, Z., Li, N., 2019. An overview on the reuse of waste glasses in alkaliactivated materials. Resour. Conserv. Recycl. 144, 297-309. https://doi.org/10.1016/j.resconrec.2019.02.007

Luukkonen, T., Abdollahnejad, Z., Yliniemi, J., Kinnunen, P., Illikainen, M., 2018. One-part alkali-activated materials: A review. Cem. Concr. Res. https://doi.org/10.1016/j.cemconres.2017.10.001

Mejía, J.M., Mejía De Gutiérrez, R., Montes, C., 2016. Rice husk ash and spent diatomaceous earth as a source of silica to fabricate a geopolymeric binary binder. J. Clean. Prod. 118, 133-139. https://doi.org/10.1016/j.jclepro.2016.01.057

Mohan, S.V., Katakojwala, R., 2021. The circular chemistry conceptual framework: A way forward to sustainability in industry 4.0, Current Opinion in Green and Sustainable Chemistry, 28, 100434. https://doi.org/10.1016/j.cogsc.2020.100434.

Moraes, J.C.B., Font, A., Soriano, L., Akasaki, J.L., Tashima, M.M., Monzó, J., Borrachero, M. V., Payá, J., 2018. New use of sugar cane straw ash in alkali-activated materials: A silica source for the preparation of the alkaline activator. Constr. Build. Mater. 171, 611-621. https://doi.org/10.1016/j.conbuildmat.2018.03.230

Pavía, S., Caro, S., 2008. An investigation of Roman mortar technology through the petrographic analysis of archaeological material. Cons. Build. Mat. 22, 1807-1811. https://doi.org/10.1016/j.conbuildmat.2007.05.003.

Payá, J., Monzó, J., Roselló, J., Borrachero, M.V., Font, A., Soriano, L., 2020. Sustainable soilcompacted blocks containing blast furnace slag (BFS) activated with olive stone biomass ash (OBA). Sustain. 12, 1-15. https://doi.org/10.3390/su12239824 
Peys, A., Rahier, H., Pontikes, Y., 2016. Potassium-rich biomass ashes as activators in metakaolinbased inorganic polymers. Appl. Clay Sci. 119, 401-409. https://doi.org/10.1016/j.clay.2015.11.003

Phair, J. W., 2006. Green chemistry for sustainable cement production and use. Green chemistry, 8(9), 763-780.

Rajan, H.S., Kathirvel, P., 2021. Sustainable development of geopolymer binder using sodium silicate synthesized from agricultural waste. J. Clean. Prod. 286, 124959. https://doi.org/10.1016/j.jclepro.2020.124959

Roselló, J., Soriano, L., Santamarina, M.P., Akasaki, J.L., José Luiz P. Melges, J.L.P., Payá, J., 2015. Microscopy characterization of silica-rich agrowastes to be used in cement binders: bamboo and sugarcane leaves. Microsc. Microanal. 21, 1314-1326. doi:10.1017/S1431927615015019

Rouseková, I., Bajza, A., Živica, V., 1997. Silica fume-basic blast furnace slag systems activated by an alkali silica fume activator. Cem. Concr. Res. 27, 1825-1828. https://doi.org/10.1016/S00088846(97)00191-9

Soriano, L., Font, A., Tashima, M.M., Monzó, J., Borrachero, M.V., Payá, J., 2020. One-part blast furnace slag mortars activated with almond-shell biomass ash: A new 100\% waste-based material. Mater. Lett. 272, 127882. https://doi.org/10.1016/j.matlet.2020.127882

Tchakouté, Hervé Kouamo, Rüscher, C.H., Hinsch, M., Djobo, J.N.Y., Kamseu, E., Leonelli, C., 2017. Utilization of sodium waterglass from sugar cane bagasse ash as a new alternative hardener for producing metakaolin-based geopolymer cement. Chemie der Erde 77, 257-266. https://doi.org/10.1016/j.chemer.2017.04.003

Tong, K.T., Vinai, R., Soutsos, M.N., 2018. Use of Vietnamese rice husk ash for the production of sodium silicate as the activator for alkali-activated binders. J. Clean. Prod. 201, 272-286. https://doi.org/10.1016/j.jclepro.2018.08.025

Vassilev, S. V., Baxter, D., Andersen, L.K., Vassileva, C.G., 2010. An overview of the chemical composition of biomass. Fuel 89, 913-933. https://doi.org/10.1016/j.fuel.2009.10.022

Villca, A.R., Soriano, L., Font, A., Tashima, M.M., Monzó, J, Borrachero, M.V., Payá, J., 2021. Lime/pozzolan/geopolymer systems: Performance in pastes and mortars. Cons. Build. Mat. 276 122208. https://doi.org/10.1016/j.conbuildmat.2020.122208

Vinai, R., Ntimugura, F., Cutbill, W., Evans, R., 2021. Production of sodium silicate from bamboo leaf ash for alkali activation of binders. Open Research Exeter. https://ore.exeter.ac.uk/repository/bitstream/handle/10871/125925/Vinai\%20et\%20al.pdf?sequen $\mathrm{ce}=1 \&$ isAllowed $=\mathrm{y}$

Weil, M., Dombroswski, K., Buchwald, A., 2009. Life-Cycle analysis of geopolymers. In: Provis and van Deventer (ed), Geopolymers Structures, Processing, Properties and Industrial Applications. https://doi.org/10.1533/9781845696382.2.194 